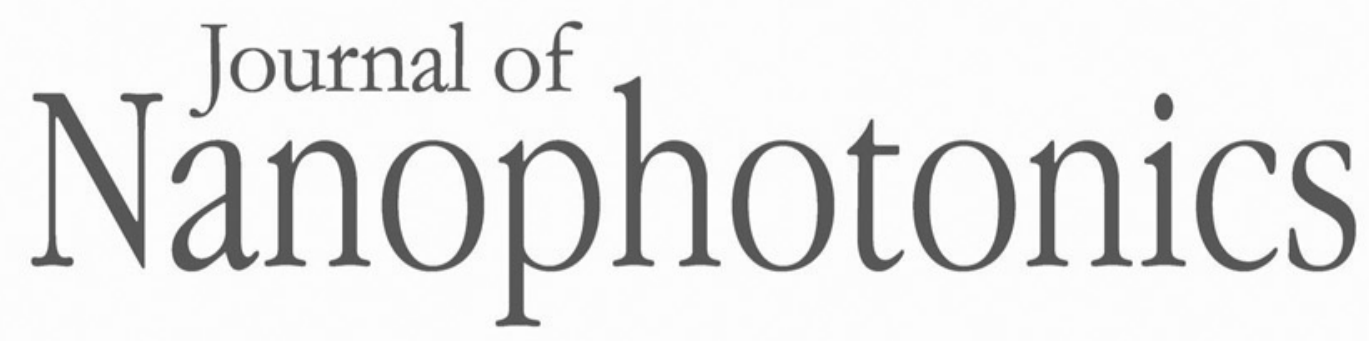

\title{
In-situ fabrication of gold nanoparticle functionalized probes for tip-enhanced Raman spectroscopy by dielectrophoresis
}

\author{
Claire S. Sweetenham \\ Richard A. J. Woolley \\ Ioan Notingher
}




\title{
In-situ fabrication of gold nanoparticle functionalized probes for tip-enhanced Raman spectroscopy by dielectrophoresis
}

\author{
Claire S. Sweetenham, Richard A. J. Woolley, and Ioan Notingher* \\ University of Nottingham, School of Physics and Astronomy, University Park, \\ Nottingham NG7 2RD, United Kingdom
}

\begin{abstract}
We report the use of dielectrophoresis to fabricate in-situ probes for tip-enhanced Raman spectroscopy (TERS) based on Au nanoparticles. A typical conductive atomic force microscope (AFM) was used to functionalize iridium-coated conductive silicon probes with Au nanoparticles of $10-\mathrm{nm}$ diameter. Suitable TERS probes can be rapidly produced (30 to $120 \mathrm{~s})$ by applying a voltage of $10 \mathrm{Vpp}$ at a frequency of $1 \mathrm{MHz}$. The technique has the advantage that the Au-based probes are ready for immediate use for TERS measurements, minimizing the risks of tip contamination and damage during handling. Scanning electron microscopy and energy dispersive $\mathrm{x}$-ray spectroscopy were used to confirm the quality of the probes, and used samples of p-ATP monolayers on silver substrates were used to demonstrate experimentally TERS measurements. ( $)$ The Authors. Published by SPIE under a Creative Commons Attribution 3.0 Unported License. Distribution or reproduction of this work in whole or in part requires full attribution of the original publication, including its DOI. [DOI: 10.1117/1.JNP.10.030502]
\end{abstract}

Keywords: tip-enhanced Raman spectroscopy; dielectrophoresis; gold nanoparticles.

Paper 16096L received Jun. 10, 2016; accepted for publication Jul. 25, 2016; published online Aug. 18, 2016.

\section{Introduction}

Tip-enhanced Raman spectroscopy (TERS) is a powerful tool for nanoscale chemical analysis. ${ }^{1,2}$ This Raman spectroscopy technique is based on the same mechanism as the surface-enhanced Raman spectroscopy (SERS) effect; ${ }^{3}$ local electric fields are strongly enhanced in the vicinity of a metallic surface due to the coupling of its plasmon resonances with the excitation light. In TERS, this metallic surface takes the form of a sharp metal tip, usually an atomic force microscopy (AFM) or scanning tunneling microscopy probe coated in silver or gold. One of the key features of TERS is that chemical information can be obtained without requiring any special sample preparation, meaning the sample can be measured in situ. TERS can achieve excellent spatial resolution, which, in principle, is only limited by the size and shape of the scanning probe microscopy (SPM) probe tip apex. The setup of TERS also ensures there is no variation of enhancement across a sample and automatically allows correlation of surface topography with chemical information.

As is the case with SERS, the development of the metallic surface that provides the enhancement of Raman scattering is essential to the success of TERS and must be optimized to realize the full potential of the technique. Commonly, there are two main approaches to produce TERS-active probes: either creation of an SPM tip by electrochemical etching of a thin wire $^{4,5}$ or modification of an existing SPM probe by metal evaporation or nanostructuring. ${ }^{6,7}$ More recently, dielectrophoresis (DEP) has been proposed for manipulation of single silver nanoparticles at the apex of AFM tips and produce TERS probes. ${ }^{8,9}$ However, the main limitation of these offline techniques is that the apex geometry is often difficult to control and the delay between fabrication and use often leads to tip contamination, oxidation, and mechanical damage (in particular for silver tips). In-situ fabrication techniques based on laser-induced synthesis of

*Address all correspondence to: Ioan Notingher, E-mail: ioan.notingher@nottingham.ac.uk 
nanoparticles at the apex of AFM tips have been reported, ${ }^{10,11}$ but these techniques have not provided sufficient reliability to allow reproducible TERS.

Here, we report the use of DEP to deposit gold nanoparticles at the AFM tip apex rapidly and in a reproducible manner. In DEP, electric field gradients can be used to manipulate and guide the growth of metal nanoparticles. ${ }^{8,9}$ The strength of the force from which the effect arises can be finely tuned since it depends on the medium, the shape, size and electrical properties of the particles, and the frequency of the electric field. The fabrication of the gold TERS tips by DEP was carried out directly on a conductive AFM; therefore, TERS measurements can, in principle, be performed immediately, thus minimizing the risks of contamination, mechanical, and electrostatic damage during handling.

\section{Experimental Methods}

A typical conductive AFM setup was used to functionalize iridium-coated conductive silicon probes with a resonant frequency of 45 to $95 \mathrm{kHz}$, spring constant of 0.5 to $4.4 \mathrm{~N} / \mathrm{m}$, and manufactured tip radius of $23 \pm 10 \mathrm{~nm}$ (MFP-3D, Asylum Research) (Fig. 1). The surface for experiments was a $1 \mathrm{~cm}^{2}$ piece of highly ordered pyrolytic graphite that was freshly cleaved and placed into a sample mount with electrical contacts. The AFM probe was loaded into a holder containing an in-built transimpedance amplifier of $20 \mathrm{nA} / \mathrm{V}$ gain with a $50 \mathrm{M} \Omega$ feedback resistor, which can measure the current at the probe-sample junction by biasing the sample with a suitable potential.

This holder was connected to the graphite and sample mount to form a circuit. After tuning the cantilever and approaching the surface, the tip was calibrated for force and electrical measurements. A small O-ring was placed in the middle of the graphite and filled with a suspension of 10-nm diameter thiol-passivated gold nanoparticles in water, forming a meniscus. The vertical position of the tip was set to the point just before the deflection started to increase and the tip was about 50 to $100 \mathrm{~nm}$ above the surface of the graphite. The frequency generator was then set to the desired frequency and voltage, which was applied over a certain time period to establish DEP between the graphite surface and the tip apex of the AFM probe.

TERS measurements were performed on a purpose-built instrument consisting of an inverted Raman microscope (Olympus IX71) and integrated AFM with a piezoelectric xy-stage (Nanowizard II, JPK Instruments). ${ }^{12}$ The instrument was equipped with a $20 \mu \mathrm{W} 532 \mathrm{~nm}$ continuous wave laser (Laser 2000, UK), a water-immersion 1.2 NA 60× objective (Olympus), and a double Czerny-Turner Raman spectrometer with a $1800 \mathrm{~L} / \mathrm{mm}$ ruled grating and a backilluminated CCD (Andor Technologies, UK).

p-ATP monolayers were deposited onto glass coverslips coated in a thin film of silver. First, the glass coverslips (VWR International) were cleaned with a variety of solvents and etched with

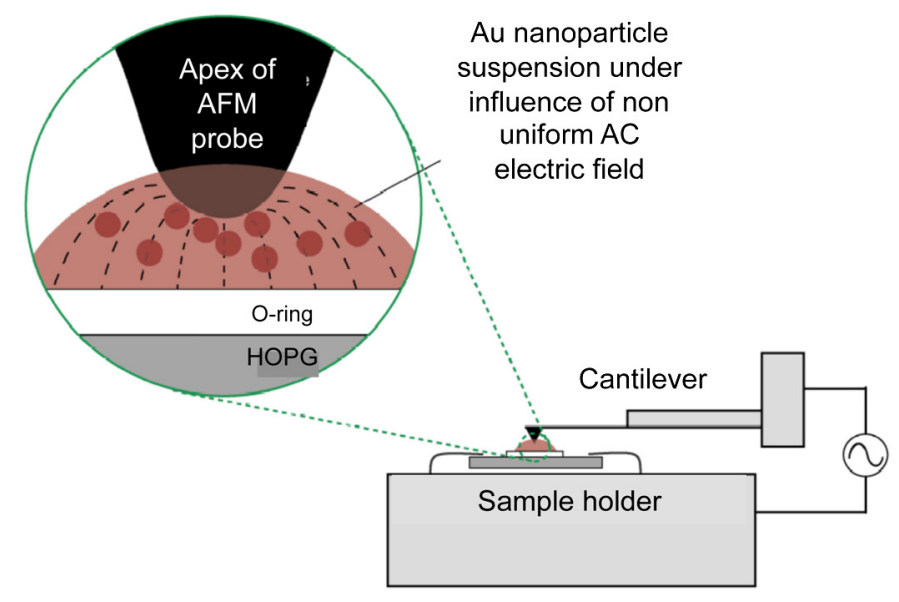

Fig. 1 Schematic of the conductive AFM setup used to functionalize probes using DEP (detection and feedback mechanisms omitted from the diagram). 
a piranha solution of 3:1 sulfuric acid and hydrogen peroxide $\left(\mathrm{H}_{2} \mathrm{SO}_{4}: \mathrm{H}_{2} \mathrm{O}_{2}\right)$ (Sigma Aldrich, UK) for hydrophilicity. Then a $\sim 25 \mathrm{~nm}$ thin film of silver was thermally evaporated in vacuum (Edwards) onto these coverslips at a pressure of 10 to $7 \mathrm{mbar}$ and evaporation rate of 0.3 to $0.4 \mathrm{~nm} / \mathrm{s}$. The coated coverslips were immersed in 10-4M p-ATP methanol solution for $2 \mathrm{~h}$ and then rinsed in methanol to ensure one molecular layer was adsorbed. One of these coverslips was then placed into the combined AFM and Raman spectroscopy system along with the AFM probe to be investigated.

\section{Results and Discussion}

During DEP, clusters of gold nanoparticles were deposited on both the graphite surface and the AFM tip. The deposition of gold nanoparticles on the surface can be used as an indication of nanoparticle cluster growth on the probe. This was evidence of DEP being generated, as material was being trapped between the tip of the AFM probe and the graphite surface. The voltage and frequency being applied to the system were tuned to deposit a small amount of material on the surface at the exact position of the tip (Fig. 2).

The frequency of $1 \mathrm{MHz}$ and voltage of $10 \mathrm{Vpp}$ was found to produce suitable, reproducible results. In addition, the size of deposition was able to be controlled by varying the period of time over which the voltage was applied to the system, from around 30 to $120 \mathrm{~s}$. This growth on the graphite suggested that nanoparticles were collecting and aggregating between the tip apex and the surface, and then breaking apart to form structures on each. This was the clearest indication from any AFM measurements that DEP was being established and reproducibly functionalizing the probes inserted into the setup. When higher frequencies and voltages of around $15 \mathrm{MHz}$ and $20 \mathrm{Vpp}$ were applied to the system, large differences in the force curves recorded before
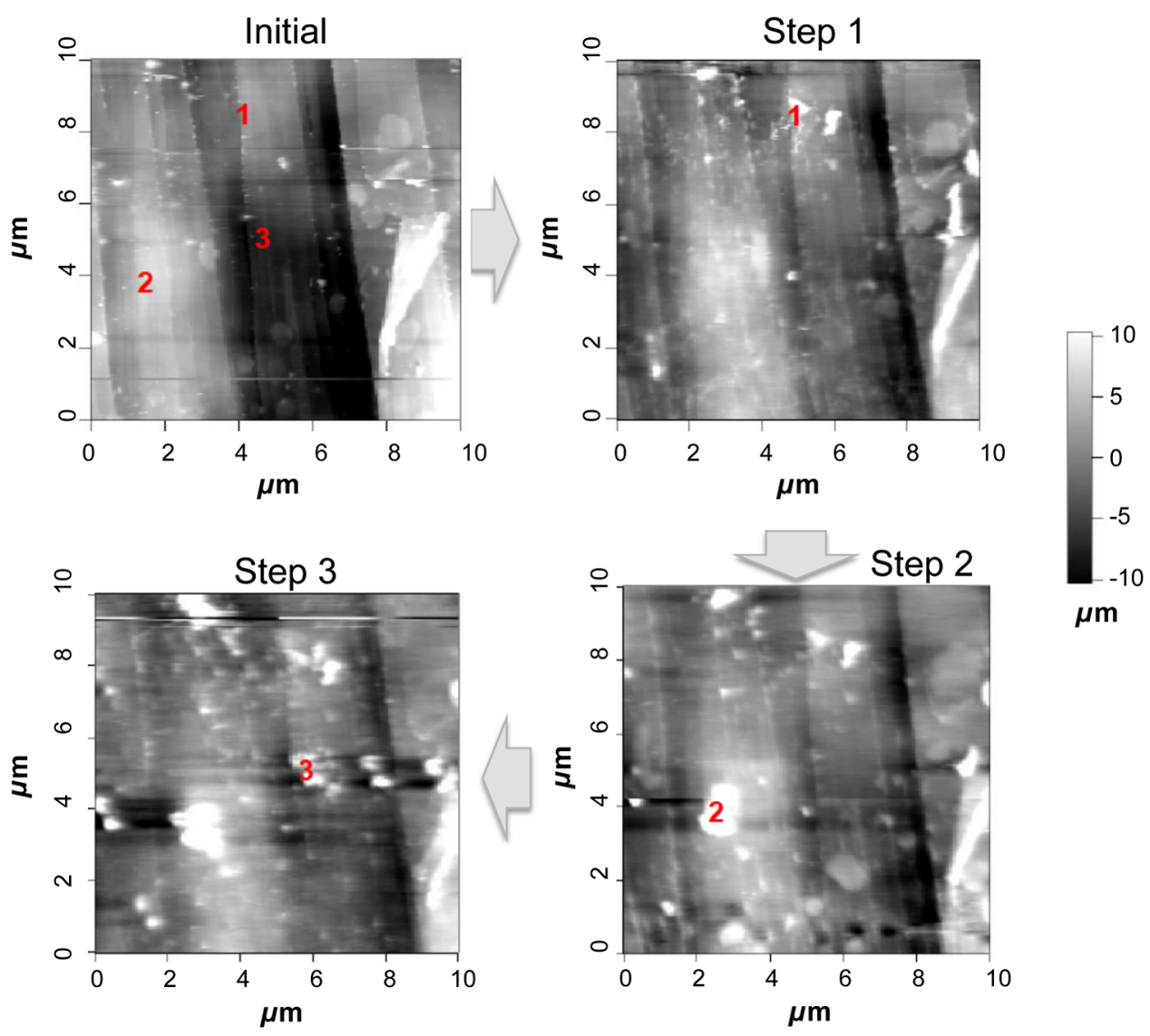

Fig. 2 Successive AFM images show the growth of nanoparticle clusters on the graphite surface after applying a voltage of $10 \mathrm{Vpp}$ at $1 \mathrm{MHz}$ for $30 \mathrm{~s}$ at position 1 (step 1), for $60 \mathrm{~s}$ at position 2 (step 2), and then for $30 \mathrm{~s}$ at position 3 (step 3). 
and after applying these voltages indicated that a large amount of material had been added to the tip of the AFM probe (not shown). These force spectroscopy results were supported by a number of imaging parameters that were monitored over the course of these experiments. There was a large variation in the deflection of the cantilever and the vertical position of the tip, which suggested a reduction in the distance between the tip and the surface of the graphite. There was a significant increase of about $0.16 \mathrm{~N} / \mathrm{m}$ in the spring constant of the AFM cantilever, indicating that the probe had gained mass.

The probes fabricated at $10 \mathrm{Vpp}$ and $1 \mathrm{MHz}$ were capable of scanning the surface of graphite and producing reasonable topographical images of it [Fig. 3(a)]. There was an obvious deterioration in imaging quality before and after performing each DEP experiment as material (Au nanoparticles) had accumulated and been added to the surface. The AFM probes functionalized with DEP were analyzed with scanning electron microscopy (SEM) and energy dispersive x-ray spectroscopy (EDX) [Figs. 3(b) and 3(c)]. The AFM probes exposed to DEP had a significant clustering of nanoparticles at the tip apex. The chemical nature of this growth was analyzed quantitatively with EDX; alongside the expected elemental composition of an AFM probe, spectral data revealed an abundance of gold within the region of the tip apex (5\% to 10\% weight). This analysis confirmed that the growth consisted of Au nanoparticles, assembled from suspension at the tip apex of the AFM probe by DEP. It is the location of this growth that makes these probes suitable for TERS studies.

(a)

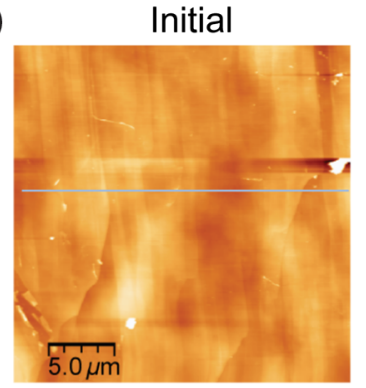

After DEP

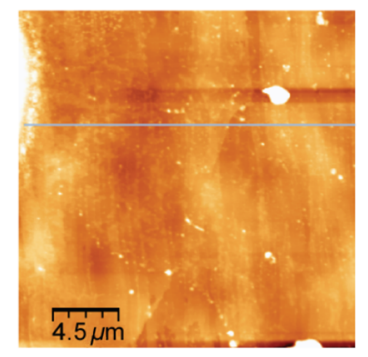

(b)

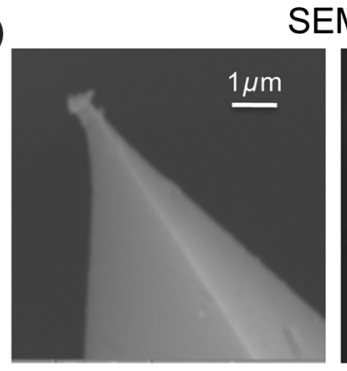

SEM

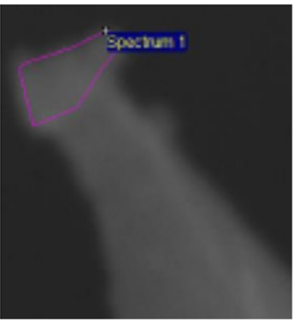

(c)

\begin{tabular}{cccccr}
\hline Element & App. conc. & Intensity corrn. & Weight \% & Weight \% sigma & Atomic \% \\
\hline C K & 4.73 & 0.2607 & 20.79 & 1.72 & 39.54 \\
O K & 1.95 & 0.6328 & 3.52 & 0.59 & 5.03 \\
Al K & 56.02 & 1.0740 & 59.73 & 1.44 & 50.58 \\
Si K & 1.17 & 0.5581 & 2.40 & 0.23 & 1.95 \\
Fe K & 0.53 & 0.9213 & 0.66 & 0.14 & 0.27 \\
Cu K & 3.64 & 0.8981 & 4.64 & 0.33 & 1.67 \\
Au M & $\mathbf{4 . 6 3}$ & $\mathbf{0 . 6 4 2 4}$ & $\mathbf{8 . 2 5}$ & $\mathbf{0 . 7 0}$ & $\mathbf{0 . 9 6}$ \\
& & & & & \\
totals & & & 100.00 & & \\
\hline \hline
\end{tabular}

Fig. 3 (a) AFM images and height profiles of the topography of the graphite surface before and after applying a voltage to the system. (b) SEM and EDX spectrum image of a typical TERS probe fabricated by DEP (EDX spectrum corresponds to the encircled region). (c) Atomic composition corresponding to the EDX spectrum in (b); figures for gold are highlighted in bold font. 
The TERS activity of the functionalized AFM probes was investigated by bringing the tip into contact with a monolayer of p-aminothiophenol (p-ATP) deposited on a thin silver film and measuring its Raman scattering. p-ATP is an ideal molecule for reliably determining electric field enhancement as it contains a thiol group $(-\mathrm{SH})$ that attaches to a metallic surface to form a single monolayer. Additionally, the Raman scattering of this structure is extremely weak, therefore any signal detected at low laser powers and short exposure times must arise from some form of enhancement of the scattering. However, the deposition of the p-ATP on the silver films allows higher enhancement due to the gap-mode TERS. ${ }^{13,14}$

A $500 \mathrm{~nm}^{2}$ region (similar size to the laser spot) of the coverslip was selected to record a Raman mapping. These mappings were performed to locate the position at which the tip apex of the AFM probe is aligned with the laser spot. This is essential to achieve efficient electric field enhancement and is the most challenging aspect of creating a successful TERS setup. Commonly, the probe is scanned across the laser beam while its back-scattered light is directed to the spectrometer CCD or a photodetector to be monitored. For reproducibility, the system is often aligned automatically using a piezoelectric stage and feedback mechanism. However, this setup was not possible with the instrument at the time of these experiments. Instead, the tip apex of the AFM probe was roughly aligned with the position of the laser beam using the crosshair of the optical camera. The area around this point was selected to record a chemical mapping. This time, the AFM probe was moved in a raster pattern while the sample was kept stationary [Fig. 4(a)]. Chemical mappings were constructed with a laser power of just $20 \mu \mathrm{W}$ and a step size of just $25 \mathrm{~nm}$, acquiring a Raman spectrum at each position at a rate of $5 \mathrm{~s}$ per pixel. The area of the Raman band at $1088 \mathrm{~cm}^{-1}$ ( $\mathrm{S}-\mathrm{C}$ stretching) in each spectrum was calculated after

(a)

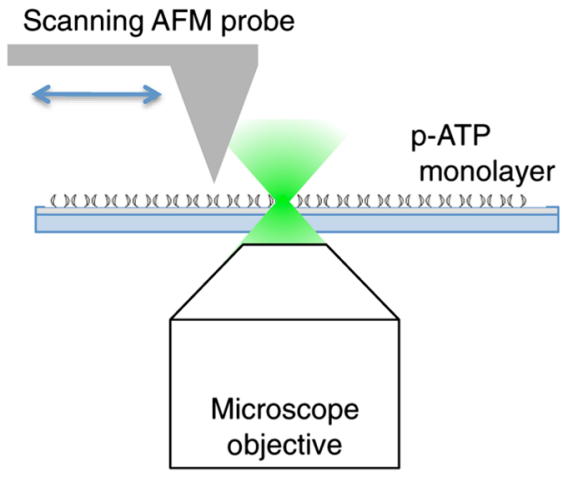

(b)

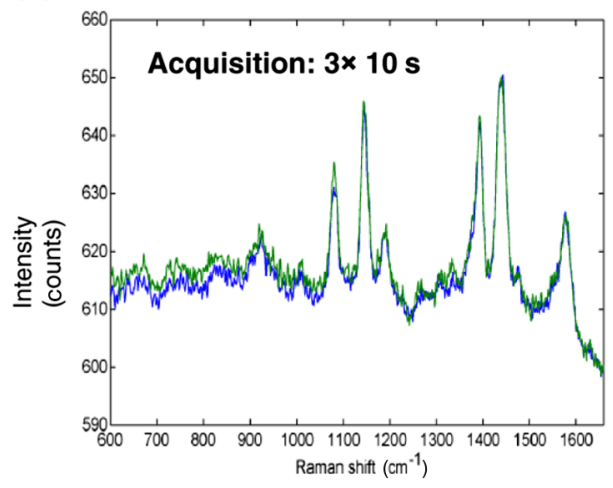

(c)

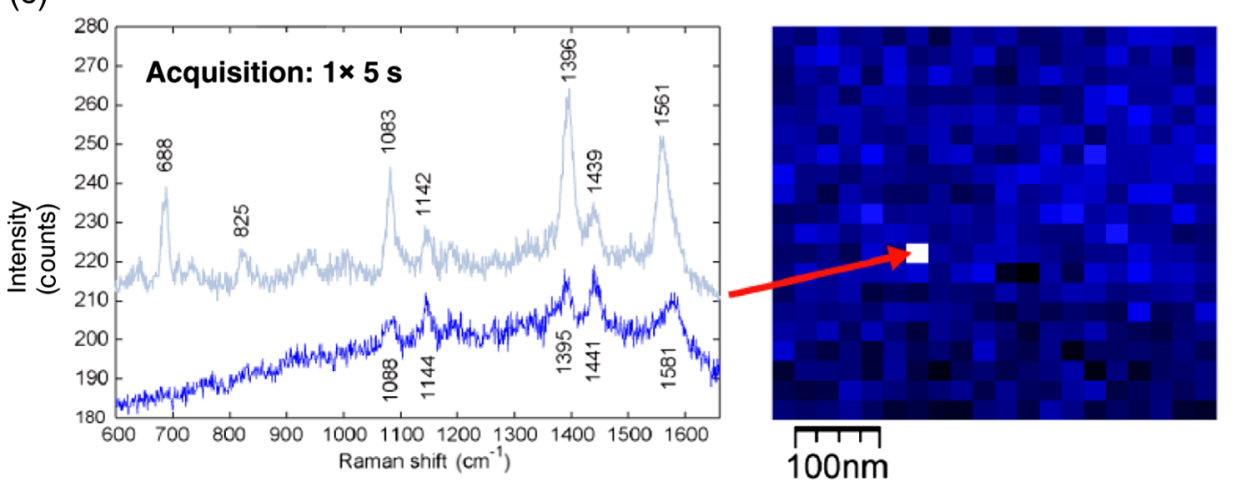

Fig. 4 (a) Schematic of TERS measurements for a p-ATP monolayer deposited on a thin silver film. The sample and laser beam are stationary and the AFM probe is scanned through the laser focus. (b) Raman spectra measured using a control AFM tip (not functionalized by DEP), both for tip retracted (green spectrum) and in contact (blue spectrum). (c) Spectra and mapping obtained with a DEP functionalized AFM probe across a p-ATP monolayer. TERS is evident in the upper trace, recorded at the corresponding position in the mapping (lower trace is a typical unenhanced Raman spectrum, which was recorded at all other positions). 
subtraction of a local linear baseline and used to build a spectral mapping 1 pixel at a time. If the AFM probe and laser spot were aligned within the area of this mapping, there would be a single pixel or small collection of pixels that were a much higher intensity than the rest.

Before taking any measurements with the functionalized AFM probe, control experiments were carried out with untreated probes [Fig. 4(b)]. Raman mappings were recorded with this probe both in contact with the p-ATP monolayer and slightly retracted, each at a rate of three accumulations of $10 \mathrm{~s}$ per pixel. This extended exposure time was required to detect the unenhanced Raman scattering that was expected from these measurements. Consequently, none of the spectra showed any sign of TERS (no individual pixel showed higher enhancement). In contrast, TERS activity was observed in Raman mappings when the functionalized AFM probes were used [Fig. 4(c)], at only $5 \mathrm{~s}$ integration time. These mappings contained a bright pixel $(25 \mathrm{~nm} \times 25 \mathrm{~nm})$, suggesting the alignment of the AFM probe with the laser beam at that position. Although the gold clusters deposited at the apex of the AFM probe were larger than the step size of $25 \mathrm{~nm} \times 25 \mathrm{~nm}$, the spatial resolution in TERS is smaller than the size of the apex, in particular, in gap-mode TERS. ${ }^{14}$ However, from analyzing the spectrum of this pixel, it could be seen that it varied from the rest of the mapping in more ways than just intensity. The Raman bands in this single spectrum are slightly shifted in frequency from their positions in the unenhanced Raman spectrum of p-ATP. The spectrum of the high intensity pixel also consists of different enhancements for different bands and contains extra bands that are not present in the spectra of the rest of the mapping. These aspects of the spectrum are consistent with those associated with SERS and TERS,${ }^{15-17}$ which arise from the variation in the selection rules of conventional Raman spectroscopy. ${ }^{18,19}$ Therefore, the combination of these spectral features with the obvious increase in the signal provides evidence for TERS and the ability of AFM probes functionalized by DEP to activate the effect. While TERS of molecular monolayers has been reported previously using tips fabricated by DEP of silver nanoparticles, this is the first report of using DEP to build TERS probes based on gold nanoparticles deposited by DEP. While gold tips have been reported to exhibit lower enhancements compared to silver probes, they have typically better stability and lower risks of contamination/oxidation compared to the silver counterparts. ${ }^{20}$

\section{Conclusions}

The potential of DEP for in-situ functionalization of AFM tips with gold nanoparticles for TERS studies has been demonstrated. These probes were developed within a conductive AFM setup consisting of a purpose-designed cantilever holder and a sample mount with electrical contacts connected in a circuit with a frequency generator. Force curves recorded before and after applying a voltage to the system indicated successful DEP. At voltages of $10 \mathrm{Vpp}$ and frequencies of $1 \mathrm{MHz}$, clusters of gold nanoparticles were deposited on the graphite surface as well as on the AFM probe. The position of these depositions was consistent with the location of the tip apex at the time of applying a voltage to the system, suggesting the trapping and growth of material between the tip and the graphite surface by DEP. It was found that these voltages and frequency over a period of 30 to $120 \mathrm{~s}$ provided suitable results for this application.

SEM and EDX analysis confirmed that the material deposited at the tip apex was a cluster of gold nanoparticles that could potentially activate TERS. These probes were assessed for TERS with the combined AFM-Raman system. Raman mappings were recorded by raster scanning the tip across the laser beam focused on a monolayer of p-ATP. The detection of an enhanced Raman spectrum only at a single point in these mappings (when the tip apex and laser were aligned within $25 \mathrm{~nm}$ ) indicated successful TERS. Further optimization of the DEP may allow fabrication of stable TERS probes with predictable signal enhancement properties.

\section{Acknowledgments}

We thank the UK Engineering and Physical Sciences Research Council (EPSRC Grant EP/P502632/1) and the Biotechnology and Biological Sciences Research Council (BBSRC Grant BB/J020877/1) for financial support. 


\section{References}

1. R. M. Stöckle et al., "Nanoscale chemical analysis by tip-enhanced Raman spectroscopy," Chem. Phys. Lett. 318(1-3), 131-136 (2000).

2. L. Langelüddecke, P. Singh, and V. Deckert, "Exploring the nanoscale: fifteen years of tip-enhanced Raman spectroscopy," Appl. Spectrosc. 69(12), 1357-1371 (2015).

3. R. Aroca, Surface-Enhanced Vibrational Spectroscopy, John Wiley and Sons Ltd., Chichester, West Sussex (2006).

4. T. Deckert-Gaudig and V. Deckert, "Tip-enhanced Raman scattering (TERS) and highresolution bio nano-analysis-a comparison," Phys. Chem. Chem. Phys. 12, 12040-12049 (2010).

5. B.-S. Yeo et al., "Tip-enhanced Raman spectroscopy_its status, challenges and future directions," Chem. Phys. Lett. 472, 1-13 (2009).

6. A. Hartschuh et al., "High-resolution near-field Raman microscopy of single-walled carbon nanotubes," Phys. Rev. Lett. 90, 095503 (2003).

7. B. Ren, G. Picardi, and B. Pettinger, "Preparation of gold tips suitable for tip-enhanced Raman spectroscopy and light emission by electrochemical etching," Rev. Sci. Instrum. 75, 837-841 (2004).

8. C. Leiterer et al., "Dielectrophoretic positioning of single nanoparticles on atomic force microscope tips for tip-enhanced Raman spectroscopy," Electrophoresis 36, 1142-1148 (2015).

9. C. Leiterer et al., "High precision attachment of silver nanoparticles on AFM tips by dielectrophoresis," Anal. Bioanal. Chem. 408, 3625-3631 (2016).

10. F. Sinjab et al., "Near-field Raman spectroscopy of biological nanomaterials by in-situ laser-induced synthesis of TERS tips," Opt. Lett. 37(12), 2256-2258 (2012).

11. T. Umakoshi et al., "Fabrication of near-field plasmonic tip by photoreduction for strong enhancement in tip-enhanced Raman spectroscopy," Appl. Phys. Express 5, 052001 (2012).

12. C. S. Sweetenham, M. Larraona-Puy, and I. Notingher, "Simultaneous SERS and AFM for label-free physicochemical analysis of lipid bilayers," Appl. Spectrosc. 65(12), 1387-1392 (2011).

13. W. Zhang et al., "Nanoscale roughness on metal surfaces can increase tip-enhanced Raman scattering by an order of magnitude," Nano Lett. 7(5), 1401-1405 (2007).

14. I. Notingher and A. Elfick, "The effect of sample and substrate electric properties on the electric field enhancement at the apex of SPM nano-tips," J. Phys. Chem. B 109, 1569915706 (2005).

15. D. Maniu et al., "Density functional theory investigation of p-aminothiophenol molecules adsorbed on gold nanoparticles," J. Optoelectron. Adv. Mater. 9(3), 733-736 (2007).

16. T. Deckert-Gaudig, E. Rauls, and V. Deckert, "Aromatic amino acid monolayers sandwiched between gold and silver: a combined tip-enhanced Raman and theoretical approach," J. Phys. Chem. C 114, 7412-7420 (2010).

17. W. Zhang et al., "Tip-enhanced Raman spectroscopy reveals rich nanoscale adsorption chemistry of 2-mercaptopyridine on Ag," Isr. J. Chem. 47, 177-184 (2007).

18. M. Moskovits and J. S. Suh, "Surface selection rules for surface-enhanced Raman spectroscopy: calculations and application to the surface-enhanced Raman spectrum of phthalazine on silver," J. Phys. Chem. 88(23), 5526-5530 (1984).

19. S. Berweger and M. B. Raschke, "Polar phonon mode selection rules in tip-enhanced Raman scattering," J. Raman Spectrosc. 40, 1413-1419 (2009).

20. L. Meng et al., "Gold-coated AFM tips for tip-enhanced Raman spectroscopy: theoretical calculation and experimental demonstration," Opt. Express 23(11), 13804-13813 (2015). 